\title{
Monte Carlo Modelling of Photodynamic Therapy Treatments Comparing Clustered Three Dimensional Tumour Structures With Homogeneous Tissue Structures
}

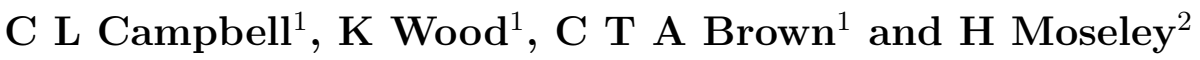 \\ ${ }^{1}$ School of Physics and Astronomy, University of St Andrews, UK \\ ${ }^{2}$ Photobiology Unit, Ninewells Hospital and Medical School, University of Dundee, \\ UK \\ E-mail: clc57@st-andrews.ac.uk
}

April 2016

\begin{abstract}
.
We explore the effects of three dimensional (3D) tumour structures on depth dependent fluence rates, photodynamic doses (PDD) and fluorescence images through Monte Carlo radiation transfer modelling (MCRT) of photodynamic therapy (PDT). The aim with this work was to compare the commonly used uniform tumour densities with non-uniform densities to determine the importance of including 3D models in theoretical investigations. It was found that fractal 3D models resulted in deeper penetration on average of therapeutic radiation and higher PDD. An increase in effective treatment depth of $1 \mathrm{~mm}$ was observed for one of the investigated fractal structures, when comparing to the equivalent smooth model. Wide field fluorescence images were simulated, revealing information about the relationship between tumour structure and the appearance of the fluorescence intensity. Our models indicate that the 3D tumour structure strongly affects the spatial distribution of therapeutic light, the PDD and the wide field appearance of surface fluorescence images.
\end{abstract}

\section{Introduction and Background}

Photodynamic therapy (PDT) utilises the interaction between light, molecular oxygen and a photosensitiser, to achieve selective tissue destruction through the production of singlet oxygen. PDT is a common method of topically treating non-melanoma skin cancer (NMSC) and precancerous lesions such as Aktinic Keratosis (AK). The non-invasive nature of the treatment and the good cosmetic outcome makes this method an attractive option when treating superficial skin lesions. For topical PDT, a cream containing either 5-aminolaevulinic acid (ALA) or its methyl ester, methyl aminolevulinate (MAL) is added to the surface of the lesion. The cream subsequently diffuses through the skin and is converted to the photosensitive molecule Protoporphyrin 
IX (PpIX). PpIX is produced at a higher rate by lesional cells, resulting in selective accumulation and localisation (Wachowska et al., 2011; Darlenski and Fluhr, 2012; Castano et al., 2004; Wilson and Patterson, 2008). PpIX absorbs light over a wide range of wavelengths, hence there is a range of different suitable light sources. The Aktilite is a red light emitting diode (LED) based light source commonly adopted for conventional PDT (Moseley, 2005), and is considered in this work.

To further investigate the light interaction with skin tissue during PDT, theoretical models are beneficial.The work presented here provides an extension of previously published work by our group (Campbell et al., 2015a). Monte Carlo radiation transfer (MCRT) modelling is commonly regarded as a useful tool for theoretical interpretation of PDT (Binzoni et al., 2008; Zhu and Liu, 2013; Swartling et al., 2003; Prahl et al., 1989). To our knowledge the majority of existing MCRT models simulating PDT assume uniform distributions of skin tissue (Zhu and Liu, 2013). Some models introduce layers of different tissue types and/or tumours represented by geometric shapes (Campbell et al., 2015a; Binzoni et al., 2008; Lister et al., 2014, 2012b; Jacques, 2010; Wang et al., 1995; Meglinski and Matcher, 2003). Three dimensional (3D) MCRT models have however previously been introduced when studying brain tissue (Boas et al., 2002) and blood vessel networks (Davis et al., 2011). Here we introduce 3D tumour structures into theoretical simulations of PDT where the mass of the tumour has been redistributed in a clustered manner. The fluence rate, photo-toxicity and surface fluorescence images are compared for different clustered structures with a corresponding smooth uniform model. Even though 3D voxel based MCRT models have been adopted to study light distribution through tissue containing different 3D structures (Pfefer et al., 1996; Patwardhan et al., 2005; Baran and Foster, 2014), to our knowledge this is the first attempt in introducing heterogeneous skin tumour tissue where the importance of including these features is explored.

\section{Methods}

MCRT modelling utilises the probabilistic nature of photon interactions to simulate scattering, absorption and propagation of light through highly scattering media. When a photon travelling through skin tissue reaches an interaction location, it can either be scattered or absorbed. The probability of the different events are determined by the optical properties (scattering and absorption coefficients) of the medium in which the photon is travelling (Prahl et al., 1989; Swartling et al., 2003; Premru et al., 2013). Due to the requirements of dealing with heterogeneous media, multiple anisotropic scattering and boundary effects, MCRT modelling is a preferred modelling technique compared to other radiation transfer techniques such as the diffusion approximation. The code that was used throughout this work was developed from a publicly available FORTRAN code (Wood, 2013; Wood and R. J. Reynolds, 1999). The original code was developed for astronomy applications, but was subsequently adapted for simulating PDT and has been extensively validated (Campbell et al., 2015a,b; Valentine et al., 2011a, 2013; Valentine, 
2011).
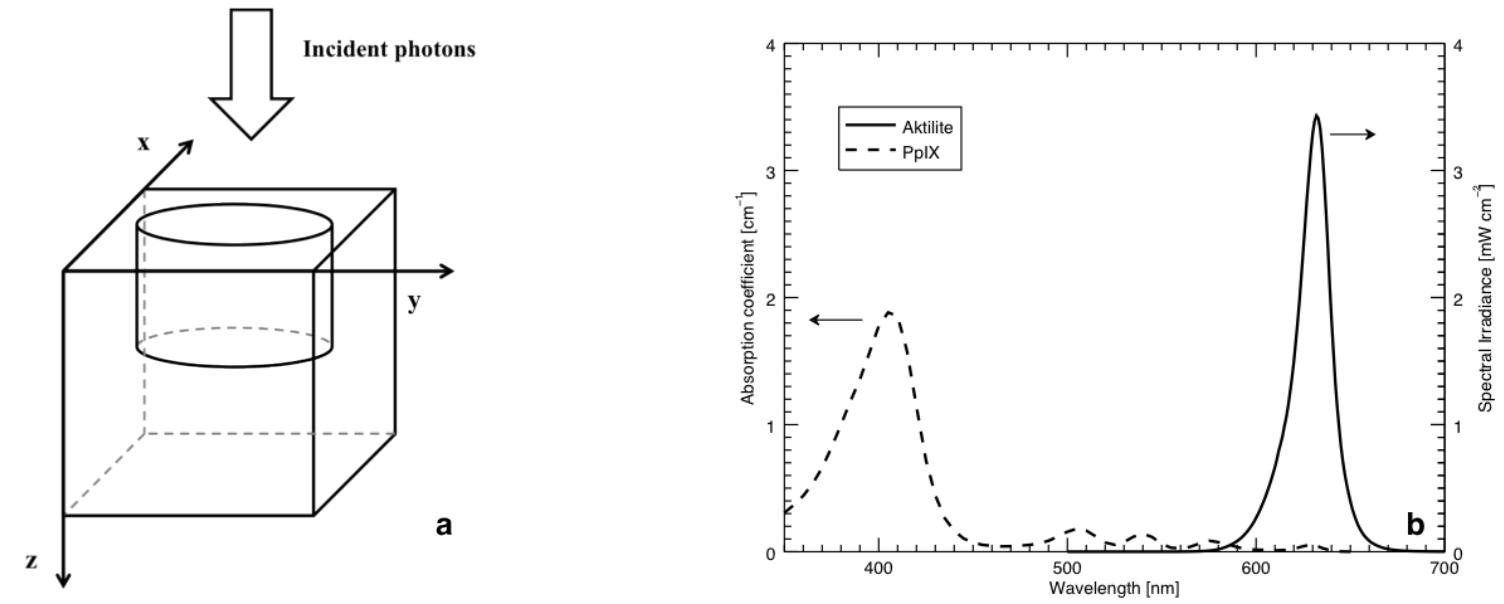

Figure 1: a)The MCRT simulation employs a 3D cartesian grid (100 grid cells on a side) which is illuminated from above. The optical properties are specified in each individual voxel. A cylindrical tumour is added in the centre of the grid, in which the PpIX is accumulated. For the non-uniform model the density of the tumour was redistributed within the cylinder. For the uniform model the density of the tumour was assumed to be uniform and the total tumour mass was assumed to be equal for all models. The concentration of the PpIX was assumed be proportional to that of the tumour. The dimensions of the grid were $10 \mathrm{~mm}$ on a side and the tumour was assumed to have a $6 \mathrm{~mm}$ diameter with a depth of $5 \mathrm{~mm}$. b) Figure showing the absorption coefficient of PpIX (dashed). The absorption coefficient is concentration dependent and for this simulation we assume an initial average number density of PpIX molecules corresponding to $7.84 \times 10^{13} \mathrm{~cm}^{-3}$.(Valentine, 2011; Valentine et al., 2013). The figure also shows the light spectrum of the Aktilite (solid), a common light source used for PDT and the light sources simulated in the work presented here. The irradiance of the Aktilite was assumed to be $82 \mathrm{~mW} \mathrm{~cm}^{-2}$.

\subsection{Code details}

By tracking power packets (hereafter referred to as photons) on their random walk, the light distribution is built up within the MCRT simulation volume. The power of the source, $P(\mathrm{~W})$, is split among $N$ Monte Carlo photons, giving each Monte Carlo photon the radiant power $P / N$. The developed code simulates the propagation of these photons through a 3D Cartesian grid containing voxels. The optical properties of each voxel may be changed independently allowing both homogeneous and inhomogeneous structures to be modelled in $3 \mathrm{D}$. The grid presented here was assumed to have the dimension of $10 \mathrm{~mm} \times 10 \mathrm{~mm} \times 10 \mathrm{~mm}$, containing $100 \times 100 \times 100$ voxels. A cylindrical 
tumour was place in the centre of the grid with a diameter of $6 \mathrm{~mm}$ and a depth of 5 $\mathrm{mm}$. The tissue surrounding the cylindrical tumour volume was assumed to be uniform and of single (dermis) tissue type. The mass of the tumour was conserved but for the heterogeneous models the mass was regrouped within the cylinder to represent different tumour structures. These will be discussed further in section 2.2.

In the code, photons are given an initial direction and wavelength and launched from the top of the grid. It was assumed that the photons normally illuminate the surface of the skin. The whole surface area of the tissue, including both healthy and tumour tissue was assumed to be uniformly illuminated. The photons are subsequently followed on their random walk through the tissue model until they are either absorbed or scattered out of the simulation grid (figure $1 \mathrm{a}$ ). Absorbed photons can be re-emitted at a different wavelength as fluorescent emission (Swartling et al., 2003; Valentine et al., 2011a; Farrell et al., 1998), allowing the simulation of the wide field fluorescence discussed in section 2.3 .

A wide range of values for the optical properties of human skin have been reported and many of these have been summarised in recent review papers (Lister et al., 2012a; Jacques, 2013). Here, the wavelength dependent scattering $\left(\mu_{s}\right)$ and absorption coefficients $\left(\mu_{a}\right)$ for skin tissue and tumour tissue are the same as those used in our previous work (Campbell et al., 2015a). The optical properties at the centre illumination wavelength $630 \mathrm{~nm}$ are summarised in table 1 . In the model presented here, the mass of the tumour tissue is redistributed. The optical properties are assumed to scale linearly with the relative redistribution. This means that the wavelength dependent scattering and absorption coefficients of the tissue will be increased in regions hosting a higher tumour density. Equally the initial distribution and thereby the optical properties of the PpIX is assumed to follow the distribution of tumour tissue.

Table 1: Optical properties at $630 \mathrm{~nm}$ for both healthy tissue, tumour tissue and PpIX. The optical properties for the extended wavelength range can be found in (Campbell et al., 2015a). The tumour optical properties presented here are the optical properties for the uniform model $\left(f_{\text {smooth }}=100 \%\right)$ and is scaled linearly with the relative mass redistribution generated by the fractal models for the non-uniform tumour models.

\begin{tabular}{lll}
\hline & $\begin{array}{l}\mu_{\mathrm{a}} \\
\left(\mathrm{cm}^{-1}\right)\end{array}$ & $\begin{array}{l}\mu_{\mathrm{s}}^{\prime} \\
\left(\mathrm{cm}^{-1}\right)\end{array}$ \\
\hline Healthy tissue & 0.7 & 36.7 \\
Tumour tissue & 2.3 & 21.2 \\
PpIX & 0.06 & - \\
\hline
\end{tabular}

The Henyey-Greenstein phase function $(\mathrm{HG}(\theta))$ is utilised within the code to model the angular scattering phase function (Prahl et al., 1989; Henyey and Greenstein, 1941; 
Jacques et al., 1987; Van Gemert et al., 1989),

$$
H G(\theta)=\frac{1}{4 \pi} \frac{1-g^{2}}{\left(1+g^{2}-2 g \cos \theta\right)^{3 / 2}}
$$

where $\theta$ is the scattering angle and $g$ is the anisotropy factor. $g$ can take values in the range $-1 \leq g \leq 1$, where $g=0$ corresponds to isotropic scattering. Skin tissue is highly forward scattering $(g>0)$ and the wavelength dependent anisotropy factor (Van Gemert et al., 1989), $g(\lambda)=0.62+0.29 \times 10^{-3} \lambda$ (with $\lambda$ in nm) was adopted here. Polarisation was not considered and the reflectance at the air/tissue interface was determined using Fresnel reflectance assuming a uniform refractive index of 1.38 for the skin.

The concentration of the photosensitiser PpIX, and thereby the associated absorption properties, affect the photodynamic reactions since the absorption of light by the PpIX is necessary for the production of singlet oxygen. The initial uniform average number density of PpIX was assumed to be $7.84 \times 10^{13} \mathrm{~cm}^{-3}$ corresponding to the absorption properties shown in figure $1 \mathrm{~b}$. It was assumed that the scattering caused by PpIX was negligible. The concentration of PpIX depletes during the illumination due to photobleaching, which depends on the fluence rate, the treatment time, the wavelength of the illuminating light as well as the initial concentration of PpIX (Jacques et al., 1993; Farrell et al., 1998; Valentine et al., 2011a) .The photobleaching was considered within the MCRT code by introducing an iterative time dynamic where $10^{6}$ photons where launched during each time step. The wavelengths of the photons were sampled such that the probability distribution function of the light spectrum (figure $1 \mathrm{~b}$ ) was reproduced. This number of photons $\left(10^{6}\right)$ was implemented to ensure that good signal to noise ratio was achieved within a reasonable simulation time. In total, 187 time steps were adopted to simulate the full $75 \mathrm{~J} \mathrm{~cm}^{-2}$ (corresponding to $15 \mathrm{~min}$ of illumination). Since the majority of the interaction occurs at the start of the treatment, the time steps were shorter at the start of the simulated treatment. At the end of each step the PpIX concentration was updated prior to launching a new set of photons. The following equation was used to update the PpIX concentration (Campbell et al., 2015a),

$$
C(x, y, z, t)=C_{0}(x, y, z) e^{-\Psi(x, y, z) t / \beta(\lambda)}
$$

where $C(x, y, z, t)$ is the local time dependent PpIX concentration, $C_{0}(x, y, z)$ is the initial concentration, and $\Psi(x, y, z)$ is the local fluence rate in $\mathrm{W} \mathrm{cm}^{-2}$ computed in the MCRT code. The wavelength dependent photobleaching dose constant, $\beta(\lambda)$ was adopted such that,

$$
\beta(\lambda)=\beta(630) \frac{\mu_{a}(630)}{\mu_{a}(\lambda)} \frac{630}{\lambda}
$$

$\mu_{\mathrm{a}}(630)$ is the absorption coefficient of PpIX at $630 \mathrm{~nm}$. $\beta(630)$ corresponds to the photobleaching dose constant at $630 \mathrm{~nm}$ and has previously been determined to be $14 \mathrm{~J} \mathrm{~cm}^{-2}$ (Valentine et al., 2011a). $\lambda$ is the wavelength of the simulated photon in $\mathrm{nm}$ and $\mu_{\mathrm{a}}(\lambda)$ corresponds to the absorption coefficient for PpIX at that wavelength. The resulting photobleaching equation assumed an unlimited oxygen supply (Valentine 
et al., 2011a; Jacques et al., 1993). The toxic threshold that is applied only considers the average number of photons required for tissue destruction.

In the MCRT model developed here, the wavelengths of the incident photons were chosen such that the probability distribution function of the irradiance, and therefore the Aktilite spectrum, is reproduced (figure $1 \mathrm{~b}$ ). The fluence rate gives the light distribution within the skin tissue during illumination and provides information about the light penetion depth. The photodynamic dose (PDD) is defined as the number of photons absorbed by the photosensitiser, per unit volume (Jacques et al., 1993). It has been suggested that the PDD is proportional to the number of singlet oxygen molecules produced and hence can be used as an indication of the efficacy of the treatment (Farrell et al., 1998). Within the model, absorption for both tissue as well as PpIX was included. However, when determining the resulting PDD, only the absorption contribution from PpIX was considered. Here we adopt a toxic threshold, indicating the number of absorptions required for effective tissue destruction. The value $8.6 \times 10^{17}$ photons $\mathrm{cm}^{-3}$ has been determined from measurements of Photofrin in liver tissue (Patterson et al., 1990), and was adopted here for illustrative purposes. To our knowledge no similar toxic threshold has been determined for PpIX and here it was assumed that this threshold is equally applicable to PpIX, however we acknowledge differences are possible between Photofrin and PpIX thresholds. This allows for comparison with previously published results (Jacques et al., 1993; Valentine et al., 2011a; Campbell et al., 2015a) as well as between the different models explored here.

The irradiance of the light source (Aktilite) was assumed to be $82 \mathrm{~mW} \mathrm{~cm}^{-2}$ and

the estimated delivered light dose was $75 \mathrm{~J} \mathrm{~cm}^{-2}$ (Valentine et al., 2011a) (corresponding to a treatment time of approximately 15 minutes).

\subsection{Tumour model}

Histological slices (Crowson, 2006) and reconstructions of 3D skin lesions such as BCC (Scheibe et al., 2010) indicate that tumour tissue does not have a uniform structure. Representations of these non-uniformities within the tumour was introduced by adopting 3D fractal clustered structures. By regrouping the mass of the tumour into clusters in a fractal manner, a 3D structure was formed. The average density over the tumour region was consistent with a smooth equivalent model with a uniform density. The structures were generated following algorithms presented by Elmegreen (Elmegreen, 1997) and is illustrated for a $2 \mathrm{D}$ case in figure 2 . To determine the density of the new tumour models, separate hierarchical levels are set up. For the first level, $N_{1}$ points are cast randomly within a 3D Cartesian grid with the $x, y$ and $z$ coordinates all taking values in the range $(0,1)$. At each subsequent level $H, N_{H}$ points are cast around each point generated at level $(H-1)$, resulting in a clustered fractal model. The distance between the points at level $H$ and $(H-1)$ decreases with increasing hierarchical level. The maximum distance from the previous level is expressed by,

$$
\pm 0.5 \Delta^{1-H}
$$


where $H$ is the hierarchal level and $\Delta$ is the dimensionless casting length which is determined through the fractal dimension such that,

$$
f_{\text {dim }}=\log _{10} N_{H} / \log _{10} \Delta
$$

where $N_{H}$ is the number of points cast at level $H$. Figure 2 demonstrates the basics of the fractal structure that is built up through this routine in $2 \mathrm{D}$. The points that are cast outside of the grid of unit dimensions are cast on the other side of the grid. If, for example a point is cast at $x=1+\varepsilon$, that point is instead given the $x$ co-ordinate $\varepsilon$, since the $\mathrm{x}$-boundary is set to be 1 . The resulting density is proportional to the total number of points at the final hierarchical level cast in each voxel. In figure 2 the number of levels is three, hence the resulting density is proportional to the number of plus signs in each pixel (here a $5 \times 5$ grid system). The $3 \mathrm{D}$ grid of unit dimensions is thereafter mapped to the desired density grid such that the dimensions correspond to the simulated grid. In the case described here the clustered grid system is mapped onto a grid system containing $100 \times 100 \times 100$ voxels. The clustered structure is further restricted to the cylindrical tumour region (figure 1 a) by eliminating points located outside of the cylindrical volume. The density within the cylinder is scaled to ensure that the total mass of the tumour is conserved.

A fraction, $f_{\text {smooth }}$ determines the minimum density within each voxel and represents the density that cannot be resolved within the grid. The remaining fraction is distributed within the clustered fractal model. Hence $\mathrm{f}_{\text {smooth }}$ determines the minimum relative density present within the tumour region. This ensures that no voxels within the tumour region contains no mass (zero density) and thereby creating a void in the simulation region. Hence the average density for a $100 \%$ smooth model (i.e no mass redistribution) and for a model with a proportion of its mass redistributed is equal.

Three different tumour models were compared where $f_{\text {dim }}$ was varied with the aim to investigate the importance of 3D effects as it changes for different degrees of clustering. A larger $f_{\text {dim }}$ results in a wider spread of the points cast at the final level of the fractal model. A smaller $f_{\text {dim }}$ results in a tighter fractal structure containing smaller regions of higher densities. The different parameters associated with the fractal models are summarised in table 2 . 


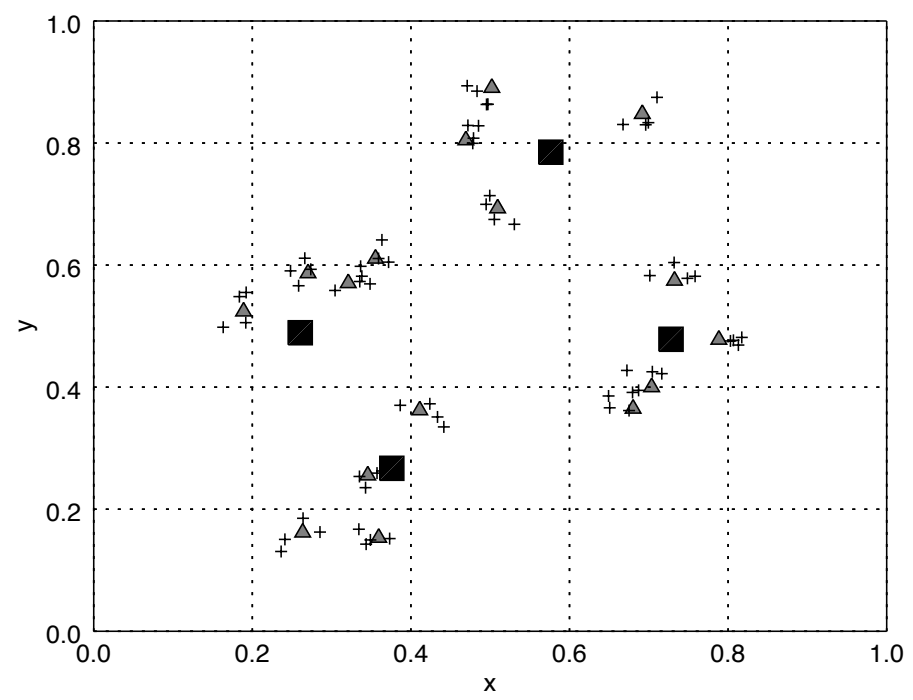

Figure 2: Figure demonstrating the clustered fractal model in 2D. Three levels are generated where the first level (squares) cast four random points within the 2D plane. The subsequent level (triangles) randomly cast four points around each point generated at the previous level, hence generating 16 new points. The third level (pluses) cast four points around each point generated at the second level, hence generating 64 new points. Only the points cast at the final level contribute to the resulting density grid, hence only the number of pluses within each pixel (dashed lines) are considered (Wood et al., 2005).

Table 2: Parameters used to generate different density structures representing different tumour structures. These parameters were chosen to demonstrate the effects generated by different degrees of non-uniformity. These chosen parameters generate artificial tumour structures where the tightness of the clusters is explored.

\begin{tabular}{llllll}
\hline Fractal model & $\begin{array}{l}\text { tumour depth } \\
(\mathrm{mm})\end{array}$ & $f_{\text {smooth }}$ & $H$ & $f_{\text {dim }}$ & $\left(N_{1}, N_{2}, N_{3}, N_{4}, N_{5}\right)$ \\
\hline 1 & 5 & $25 \%$ & 5 & 3 & $(16,32,32,32,32)$ \\
2 & 5 & $25 \%$ & 5 & 2.5 & $(16,32,32,32,32)$ \\
3 & 5 & $25 \%$ & 5 & 2 & $(16,32,32,32,32)$ \\
\hline
\end{tabular}

The tumour tissue was assumed to be confined within the cylindrical tumour region and the PpIX concentration was assumed to have an initial distribution that mapped to the redistributed tumour geometry. As we have described above, in our 3D model the tumour structure has different optical properties from the surrounding healthy tissue. This includes both scattering and absorption coefficients. When we develop our fractal based tumour structures, we assume that the optical properties of the voxels 
containing tumour tissue have the properties associated with that particular tissue type. Furthermore, we have also assumed that the optical properties of the tumour tissue scale linearly with the density of the tumour tissue presented within a voxel. Due to the restricted tumour area, the first level of the fractal model was forced to only cast points within this region. The first locations $\left(x_{1}, y_{1}, z_{1}\right)$ were therefore determined as follows

$$
\begin{aligned}
& x_{1}=0.3+0.4 \xi_{1} \\
& y_{1}=0.3+0.4 \xi_{2} \\
& z_{1}=0.5+0.5 \xi_{3}
\end{aligned}
$$

where $\xi_{1}, \xi_{2}$ and $\xi_{3}$ are different random numbers, in the range $(0,1)$. This concentrated the redistribution of the density to the cylinder which can be argued to be a more realistic representation than a model where the points at the first level are randomly cast within in the entire Cartesian grid.

Figure 3 shows cross-sections in both the vertical plane and the top slice of the density structures investigated here. Even though the cross sectional image indicates a pixel-wide slice where the clustered feature of the tumour tissue is centred close to the surface, this is not true throughout the generated tumour tissue. The clusters are randomly allocated according to equations $6-8$, which results in a clustered tumour model that is distributed throughout the assumed tumour region without bias to any particular area. Figures 4 show the relative density histograms for the tumour densities. The histograms demonstrate the distribution of different densities over the whole tumour region. The minimum density within the allocated area is equal to $f_{\text {smooth }}$ (here kept at 0.25 ). The proportion of voxels with a relative density equal to $f_{\text {smooth }}$ vary for the different models explored due to the tightness of the clusters associated with different values for $f_{\text {dim }}$.

\subsection{Fluorescence}

The wide field surface fluorescence image as a result of $405 \mathrm{~nm}$ excitation was simulated to demonstrate what is commonly observed and/or measured clinically. The fluorescence photons were isotropically reemitted from the absorption location and allocated a wavelength sampled from a PpIX fluorescence spectrum characterised by two distinct peaks at $635 \mathrm{~nm}$ and $705 \mathrm{~nm}$ (Valentine et al., 2011b). Fluorescence photons exiting the surface of the grid were recorded and binned according to location of exit. A numerical aperture of 0.22 was implemented allowing only photons exiting the simulation grid within a certain cone to be included within the grid. The motivation behind using this numerical aperture was to consider the collection efficiency associated with potential measurement equipment such as an optical biopsy system (Nadeau et al., 2004). 

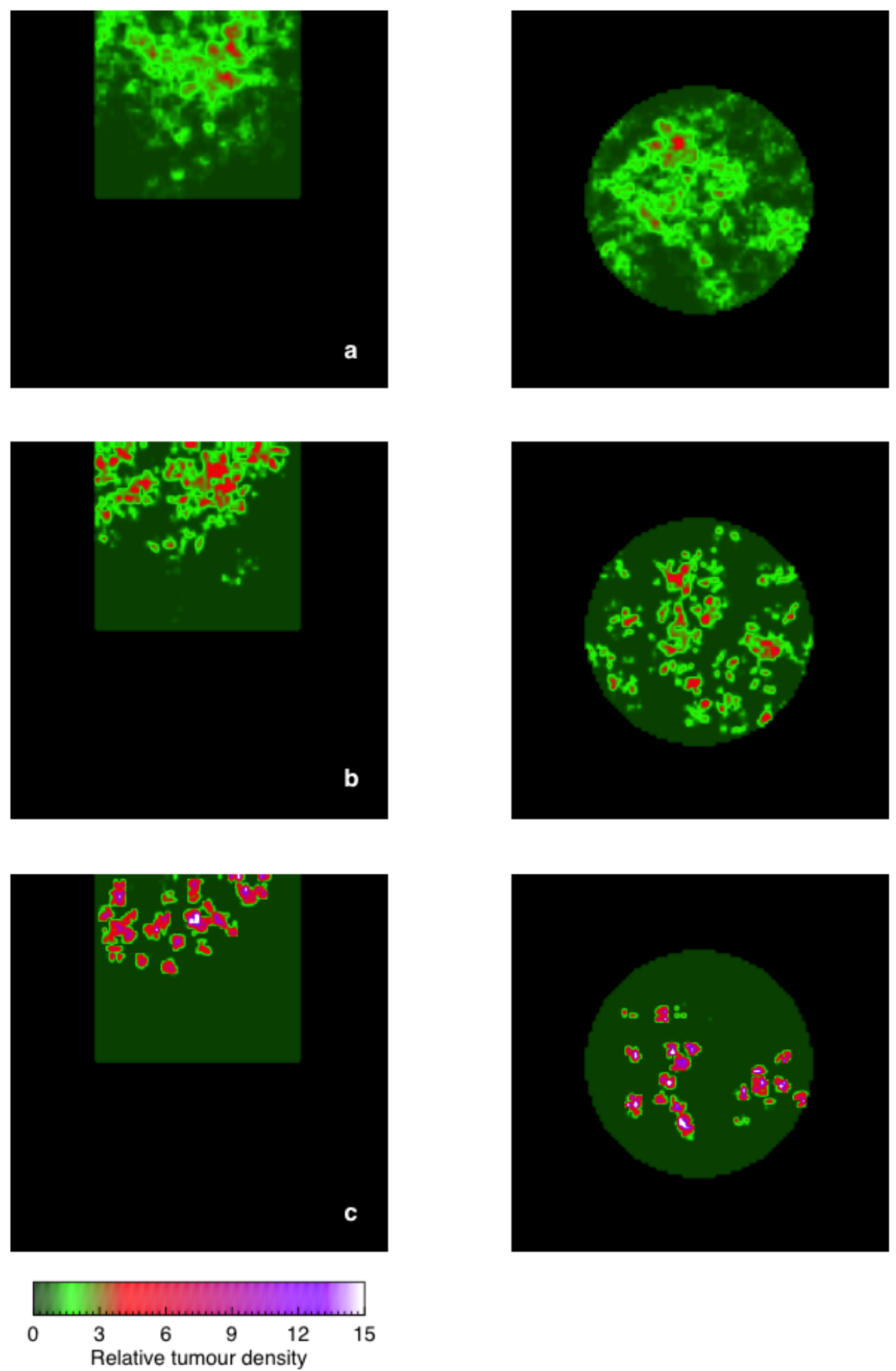

Figure 3: Figure demonstrating the density distribution of the different tumour models explored. The left column represents a pixel wide slice in the $x$ - $z$ plane in the middle of the density grid. The right column represents a pixel wide slice in the $x-y$ plane at the top of the grid. The difference in the tightness of the clusters is caused by the variation of the fractal dimensions such that a) $f_{\text {dim }}=3$, b) $f_{\text {dim }}=2.5$ and c) $f_{\text {dim }}=2$. Colour image is available in online version. 

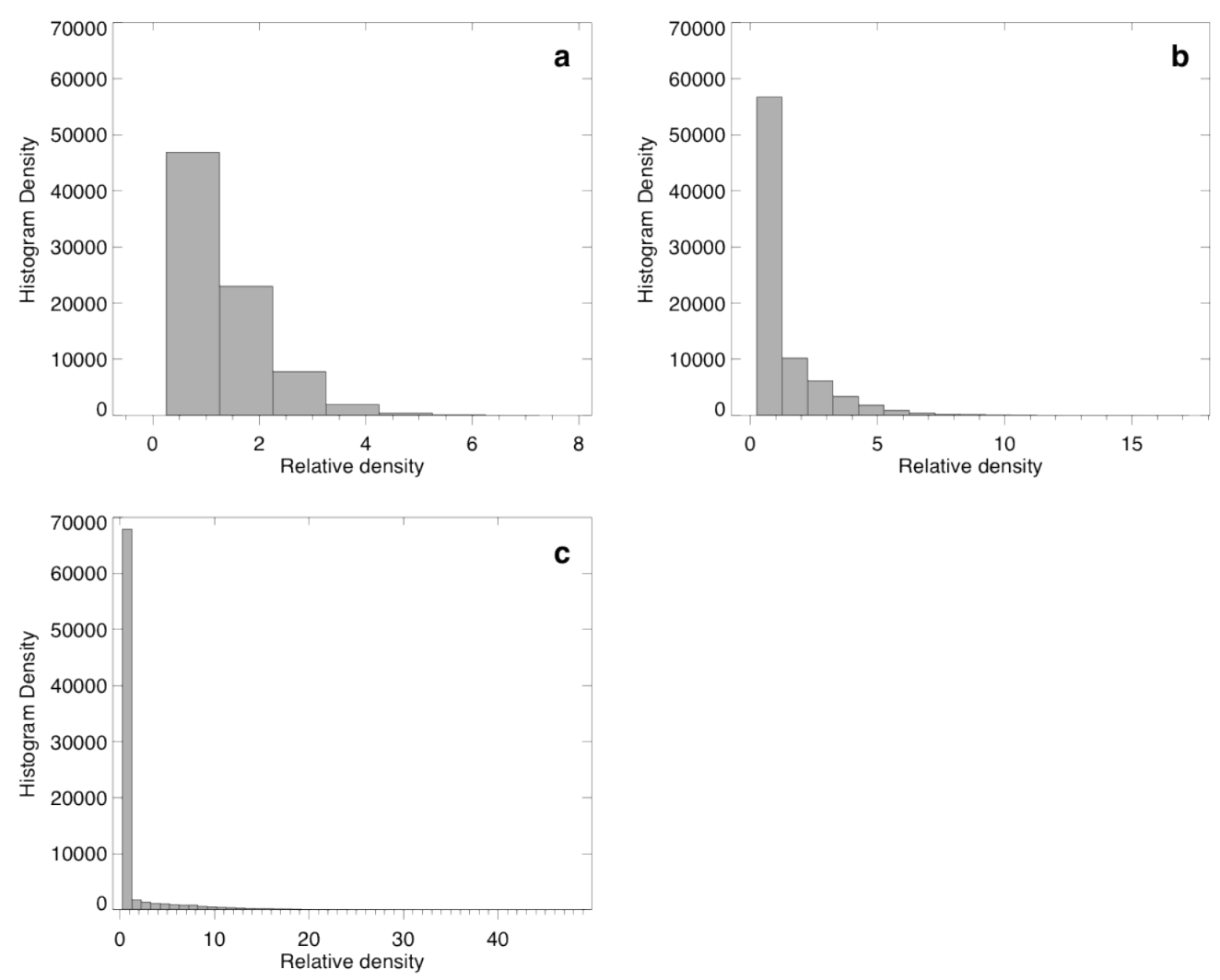

Figure 4: Figure demonstrating the density distribution of the different tumour models that were explored. The density was binned to demonstrate the distribution of different densities within different tumour structures where a) $f_{\text {dim }}=3$ and $f_{\text {smooth }}=0.25$, b) $f_{\text {dim }}=2.5$ and $f_{\text {smooth }}=0.25$ and c) $f_{\text {dim }}=2$ and $f_{\text {smooth }}=0.25$.

\section{Results}

The fluence rates for 405, 540 and $630 \mathrm{~nm}$ for the three fractal models were generated and compared to the equivalent smooth/uniform model. The resulting average fluence rates are displayed in figure 5 . All fractal models demonstrate an increase in the average fluence rate compared to the smooth model. However fractal model 3 results in a larger difference compared to fractal model 1 (table 2). This is explained by the difference in density distribution, which is demonstrated by the histograms in figure 4 . 

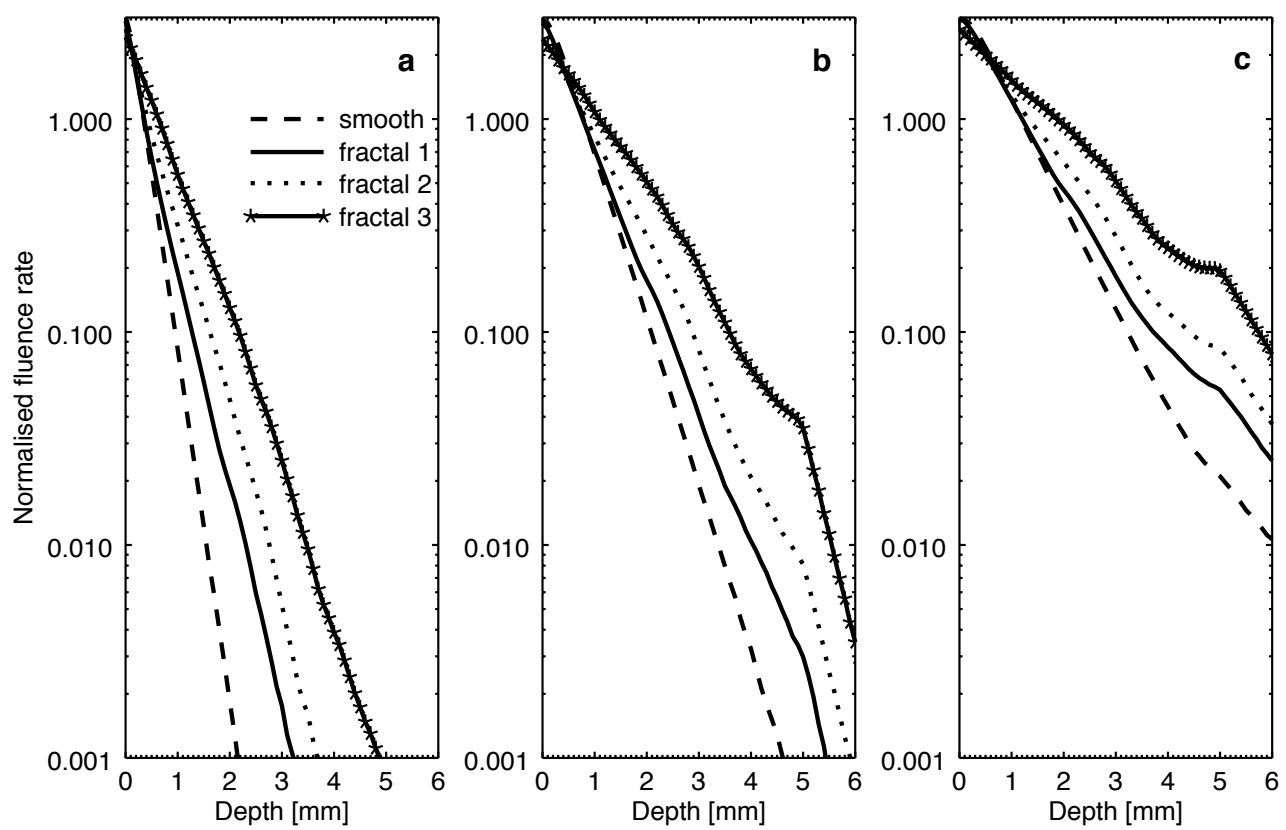

Figure 5: Average normalised fluence rate for 3D models comparing the different fractal models $\left(1: f_{\text {dim }}=3,2: f_{\text {dim }}=2.5,3: f_{\text {dim }}=2\right)$ for a) $\left.405 \mathrm{~nm} \mathrm{~b}\right) 540 \mathrm{~nm}$ and c) 630 $\mathrm{nm}$. These plots are normalised by the irradiance of the light source. The fluence rate is reduced at $5 \mathrm{~mm}$ due to the change in optical properties between the tumour tissue and the healthy tissue surrounding the tumour tissue.

The PDD for the different fractal models (table 2) as well as the corresponding smooth model, using the Aktilite as the illuminating light source is shown in figure 6 . The smooth/uniform model $\left(\mathrm{f}_{\text {smooth }}=100 \%\right)$ assumed an initial uniform distribution of the tumour/PpIX equal to the average density of the fractal models. The PDD for the smooth model (dashed line) is included in figure 6 and is equal for all three fractal structures. The figure additionally shows the average for the fractal models (solid lines) as well as individual vertical sample sections demonstrating the range of treatment depth through different parts of the tumour (grey dotted lines). From the results it is clear that fractal structure 3 (figure $6 \mathrm{c}$ ) demonstrates the largest difference in treatment depth in comparison to the smooth model. For this structure an additional average simulated treatment depth of approximately $1 \mathrm{~mm}$ was gained compared to the corresponding smooth model. This fractal model is however also associated with the largest spread when considering vertical sample sections. Here the smooth factor $\left(\mathrm{f}_{\text {smooth }}\right)$ associated with the fractal model starts to dominate since the redistributed mass is concentrated to smaller volumes, resulting in deeper light penetration through the lower density regions comprised by a relative density equal to $f_{\text {smooth }}(25 \%)$. Figure 6 a however corresponds to a fractal model where the volume containing only the smooth component (corresponding 
to those regions with minimum relative density (0.25)) makes up only a small part of the total tumour volume. This results in an average PDD as a function of depth that does not differ significantly from the corresponding smooth/uniform model. However the vertical sample sections demonstrate the range of treatment depths achieved across the tumour region. Hence even though the average PDD is not significantly different from the smooth model, a large fraction of the individual sample sections penetrate deeper into the skin. Conversely a large fraction also penetrates to a shallower depth (figure 6 a).

Figure 7 represents the PDD through a pixel wide slice in the $x$ - $y$ plane. The cross section shows where the light is absorbed and demonstrates that the light penetrates into all areas even the high density regions (figure $7 \mathrm{c}$ ).

The simulated wide field fluorescence for the three different fractal models is shown in figure 8. The relative fluorescence intensity is displayed and the image comprises fluorescence photons generated throughout the tumour that have escaped the simulated region via scattering. The fluorscence signal appears more uniform than the cross section of the tumour density shown in figure 3. The wide field fluorescence also demonstrates the variation that might occur when using a small probe to measure the fluorescence signal. The intensity of the fluorescence changes by a factor of approximately 5 over sub-mm size scales. 

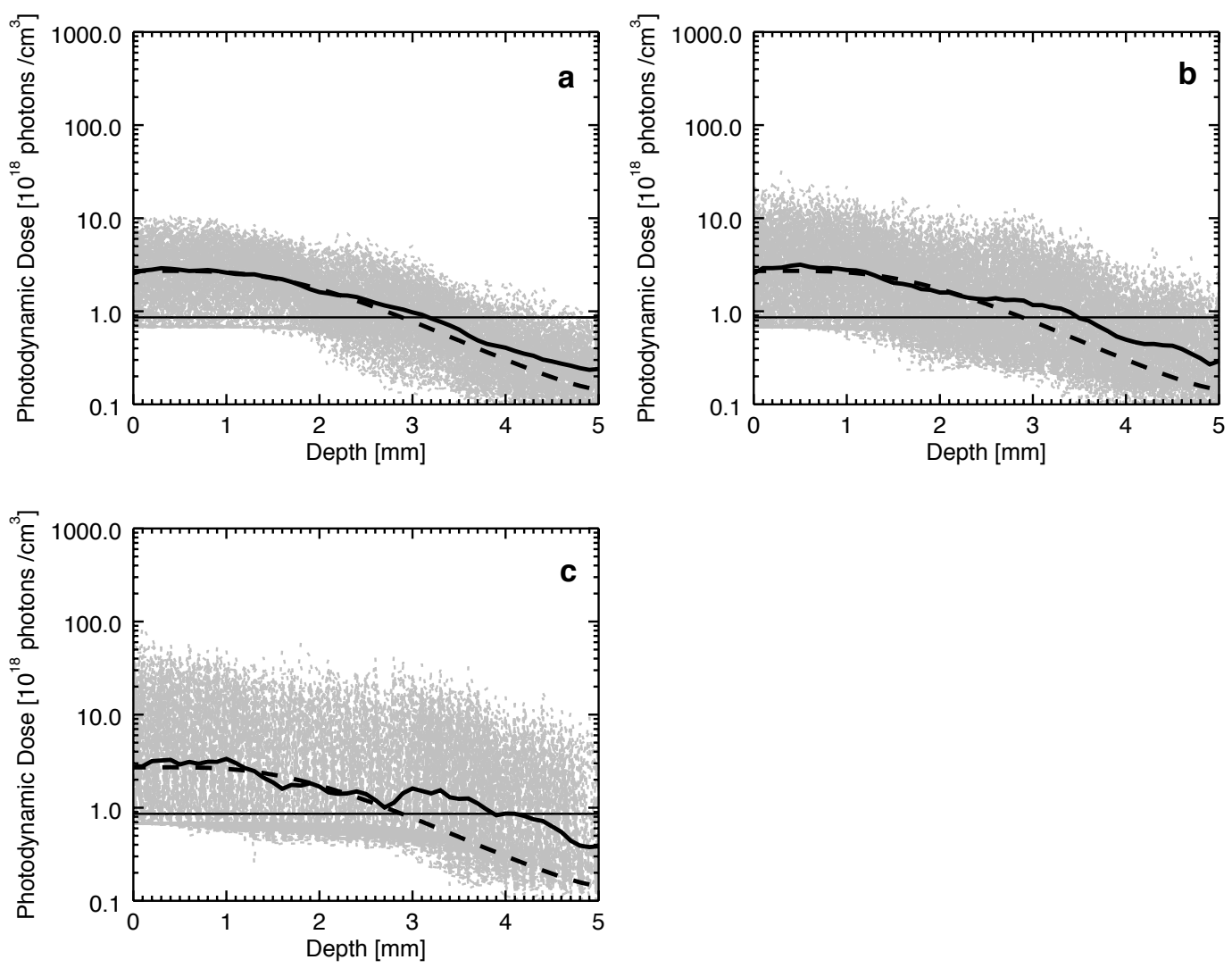

Figure 6: Photodynamic dose (PDD) for fractal structure (solid line) a ) $1\left(f_{\text {dim }}=3\right)$ b) $2\left(f_{\text {dim }}=2.5\right)$ and c $) 3\left(f_{\text {dim }}=2\right)$ as well as the smooth model (dashed line) generated by the Aktilite. Grey lines corresponds to the different vertical sample sections and demonstrate a range between the highest and the lowest PDD at the surface, which is approximately equal to a factor of a) 15, b) 30 and c) 90. The average PDD (solid line) for the fractal model is comparatively noisy in figure c due to the larger change in density in the vertical direction associated with this density structure (figure $3 \mathrm{c}$ ). In figure $c$ an increase in the simulated treatment depth of approximately $1 \mathrm{~mm}$ is noted between the uniform and the non-uniform tumour structures. 

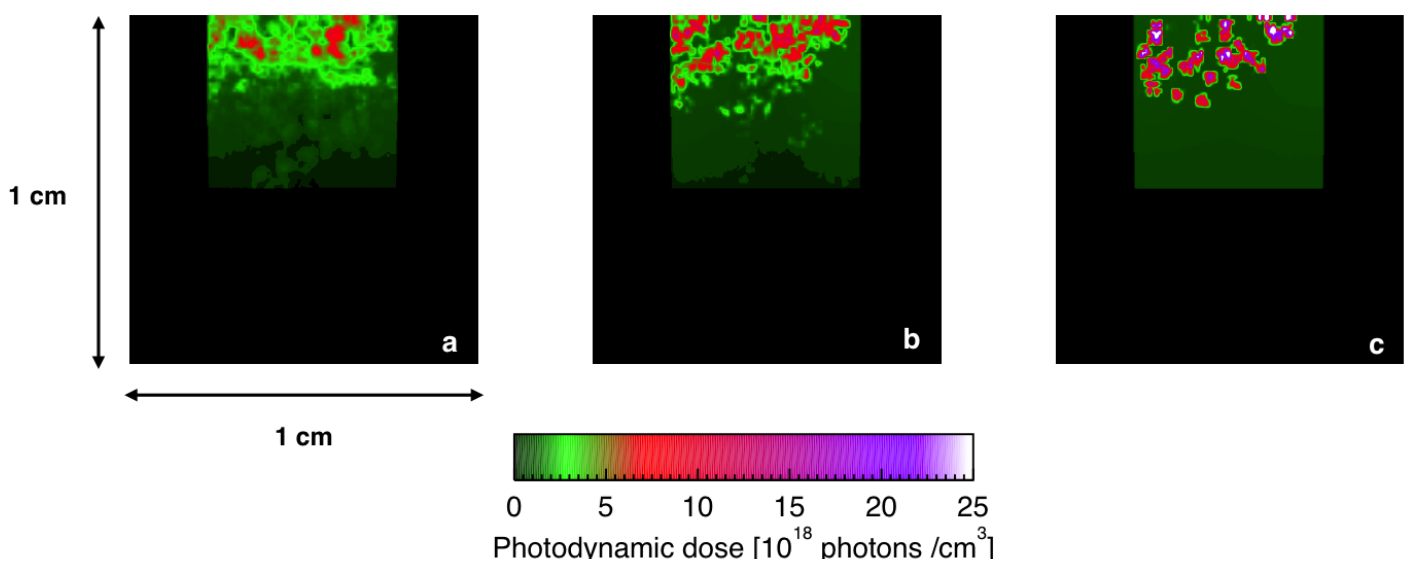

Figure 7: Cross section of the photodynamic dose (PDD) through the same plane shown in the left column of figure 3 for a) $1\left(f_{\text {dim }}=3\right)$, b) $2\left(f_{\text {dim }}=2.5\right)$ and c) $3\left(f_{\text {dim }}=2\right)$. Figure demonstrates the level of absorption after a delivered light dose of $75 \mathrm{~J} \mathrm{~cm}^{-2}$. The image dimensions are $1 \mathrm{~cm}$ on a side. Colour image is available in online version.

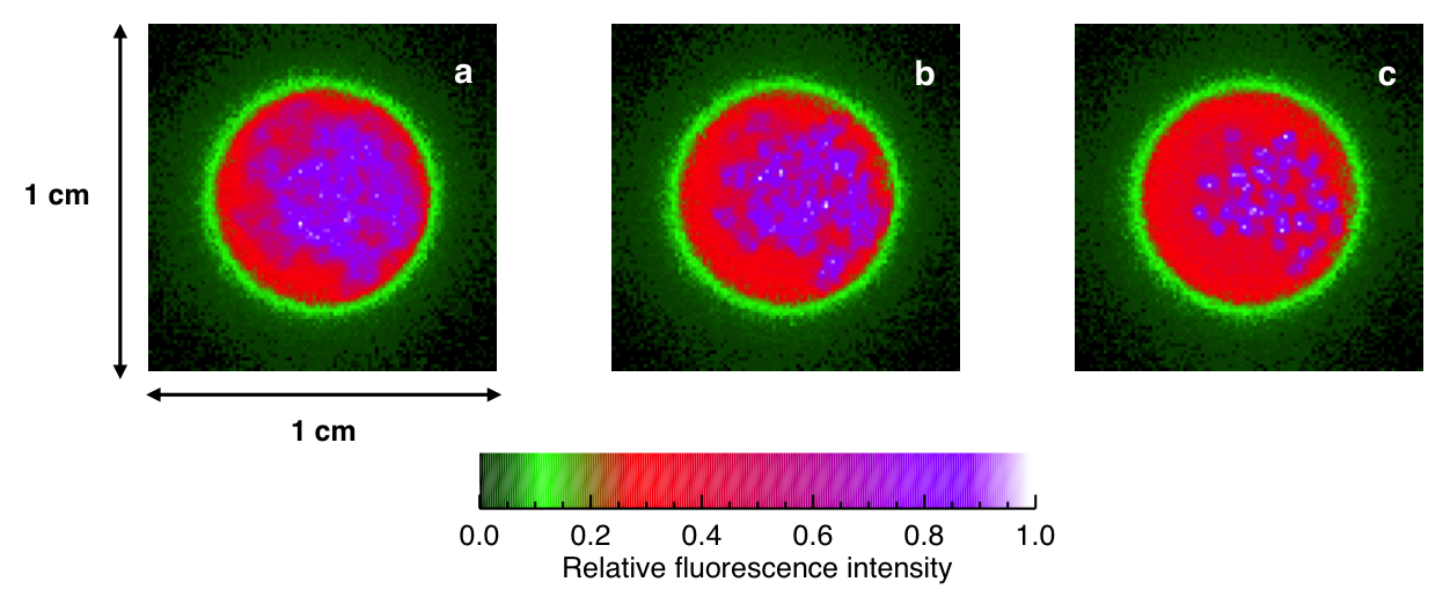

Figure 8: Figure of the simulated wide field fluorescence for fractal structures a) 1 ( $f_{\text {dim }}$ $=3)$, b) $2\left(f_{d i m}=2.5\right)$ and c) $3\left(f_{d i m}=2\right)$. The image shows both a more uniform fluorescence signal compared to a cross section of the density as well as a variation in fluorescence over small distances. The image dimensions are $1 \mathrm{~cm}$ on a side. Colour image is available in online version.

\section{Discussion}

The work presented here explores the importance of including 3D tumour structures within theoretical simulations of PDT. Most existing models only consider uniform densities of different tissue types, however here we show that tissue with a non-uniform 
distribution affects the light penetration in tissue considerably. Different degrees of redistribution of tumour mass were compared but the average density over the whole tumour region was the same for all models. The results presented in this work suggest that if there are non-uniformities within tissue, 3D effects should be considered in theoretical models.

The different vertical sample sections demonstrate the range of the penetration through different parts of the tumour. The spread increases as the smooth component, $f_{\text {smooth }}$ becomes more dominating. For a structure where the mass redistribution forms tight clusters with high density, the majority of the volume is made up of the smooth component. Hence the majority of the propagating photons will experience a medium of lower opacity which results in larger average fluence rates deeper in the tissue. The resulting regions of lower density of PpIX results in an insufficient PDD in these regions, since there are not enough absorption events possible to reach the estimated threshold. There is more absorption in regions of higher density on the other hand and consequently a higher PDD. This explains the larger spread in PDD associated with the vertical sample sections from fractal structure 3 compared to fractal structure 1 , where the density difference between regions is lower (figure 4).

The wide field fluorescence images (figure 8) comprise fluorescence light emitted not only from the surface but also contributions from the tumour below the surface. The increasing inhomogeneity of the images varies as expected between the different fractal structures. Point measurements will vary over small distances due to the clusters of higher densities.

The work presented here is to our knowledge the first to consider the effects of 3D tissue structure on light distributions during PDT. Even though the structures considered are constructed from mathematical algorithms they illustrate the effects of a non uniform distribution on the light penetration. The method of using these algorithms also allows for easy comparison to the equivalent smooth model to assess the importance of 3D affects. Future work should include more realistic tumour models perhaps based on histological samples.

\section{Conclusions}

3D tumour structures were introduced with the purpose of theoretically studying topical PDT. The effects of non-uniformities within the tissue were explored by including fractal clustered tumour models. By changing the fractal dimensions of the density structures, different degrees of redistributions were investigated. It was found that light penetration is strongly affected by the distribution of tissue and encourages continued usage of 3D MCRT models when studying light propagation through skin tissue. 


\section{Acknowledgments}

C L Campbell acknowledges financial support from an UK EPSRC PhD studentship (EP/K503162/1) and the Alfred Stewart Trust. 


\section{References}

Baran, T. M. and Foster, T. H. (2014). Comparison of flat cleaved and cylindrical diffusing fibers as treatment sources for interstitial photodynamic therapy. Medical physics, 41(2):022701.

Binzoni, T., Leung, T., Giust, R., Rufenacht, D., and Gandjbakhche, A. (2008). Light transport in tissue by $3 \mathrm{~d}$ monte carlo: Influence of boundary voxelization. Computer Methods and Programs in Biomedicine, 89(1):14 - 23.

Boas, D., Culver, J., Stott, J., and Dunn, A. (2002). Three dimensional monte carlo code for photon migration through complex heterogeneous media including the adult human head. Optics express, 10(3):159-170.

Campbell, C., Wood, K., Valentine, R., Brown, C., and Moseley, H. (2015a). Monte carlo modelling of daylight activated photodynamic therapy. Physics in medicine and biology, 60(10):4059.

Campbell, C. L., Christison, C., Brown, C. T. A., Wood, K., Valentine, R. M., and Moseley, H. (2015b). 3d monte carlo radiation transfer modelling of photodynamic therapy. In SPIE Biophotonics South America, pages 95311H-95311H. International Society for Optics and Photonics.

Castano, A., Demidova, T., and Hamblin, M. (2004). Mechanisms in photodynamic therapy: part one - photosensitizers, photochemistry and cellular localization. Photodiagnosis and Photodynamic Therapy, 1(4):279 - 293.

Crowson, A. N. (2006). Basal cell carcinoma: biology, morphology and clinical implications. Modern pathology, 19:S127-S147.

Darlenski, R. and Fluhr, J. (2012). Photodynamic therapy in dermatology: past, present, and future. Journal of Biomedical Optics, 18(6):061208-061208.

Davis, M. A., Shams Kazmi, S., Ponticorvo, A., and Dunn, A. K. (2011). Depth dependence of vascular fluorescence imaging. Biomedical optics express, 2(12):33493362 .

Elmegreen, B. G. (1997). Intercloud Structure in a Turbulent Fractal Interstellar Medium. , 477:196-203.

Farrell, T., Hawkes, R., Patterson, M., and Wilson, B. (1998). Modeling of photosensitizer fluorescence emission and photobleaching for photodynamic therapy dosimetry. Appl. Opt., 37(31):7168-7183.

Henyey, L. and Greenstein, J. (1941). Diffuse radiation in the Galaxy. , 93:70-83.

Jacques, S. (2013). Optical properties of biological tissues: a review. Physics in Medicine and Biology, 58(11):R37.

Jacques, S., Alter, C., and Prahl, S. (1987). Angular dependence of HeNe laser light scattering by human dermis. Lasers Life Sci., 1:309-333.

Jacques, S., Joseph, R., and Gofstein, G. (1993). How photobleaching affects dosimetry and fluorescence monitoring of pdt in turbid media. Proc. SPIE, 1881:168-179. 
Jacques, S. L. (2010). Optical assessment of cutaneous blood volume depends on the vessel size distribution: a computer simulation study. Journal of biophotonics, 3(1):75.

Lister, T., Wright, P., and Chappell, P. (2012a). Optical properties of human skin. Journal of Biomedical Optics, 17(9):090901-1-090901-15.

Lister, T., Wright, P., and Chappell, P. (2014). A new monte carlo program for simulating light transport through port wine stain skin. Lasers in Medical Science, 29(3):1017-1028.

Lister, T., Wright, P. A., and Chappell, P. H. (2012b). Simulating light transport through skin for color prediction of port wine stain lesions: a review. Journal of biomedical optics, 17(11):110901-110901.

Meglinski, I. and Matcher, S. (2003). Computer simulation of the skin reflectance spectra. Computer methods and programs in biomedicine, 70(2):179-186.

Moseley, H. (2005). Light distribution and calibration of commercial pdt led arrays. Photochem. Photobiol. Sci., 4:911-914.

Nadeau, V., O’Dwyer, M., Hamdan, K., Tait, I., and Padgett, M. (2004). In vivo measurement of 5-aminolaevulinic acid-induced protoporphyrin ix photobleaching: a comparison of red and blue light of various intensities. Photodermatology, Photoimmunology Photomedicine, 20(4):170-174.

Patterson, M. S., Wilson, B., and Graff, R. (1990). In vivo tests of the concept of photodynamic threshold dose in normal rat liver photosensitized by aluminum chlorosulphonated phthalocyanine. Photochemistry and Photobiology, 51(3):343-349.

Patwardhan, S. V., Dhawan, A. P., and Relue, P. A. (2005). Monte carlo simulation of light-tissue interaction: three-dimensional simulation for trans-illumination-based imaging of skin lesions. Biomedical Engineering, IEEE Transactions on, 52(7):12271236.

Pfefer, T. J., Barton, J. K., Chan, E. K., Ducros, M. G., Sorg, B. S., Milner, T. E., Nelson, J. S., and Welch, A. J. (1996). A three-dimensional modular adaptable grid numerical model for light propagation during laser irradiation of skin tissue. IEEE Journal of Selected Topics in Quantum Electronics, 2(4):934-942.

Prahl, S., Keijzer, M., Jacques, S., and Welch, A. (1989). A monte carlo model of light propagation in tissue. In SPIE Proceedings of Dosimetry of Laser Radiation in Medicine and Biology, pages 102-111. Press.

Premru, J., Milani, M., and Majaron, B. (2013). Monte carlo simulation of radiation transfer in human skin with geometrically correct treatment of boundaries between different tissues. Proc. SPIE, 8579:85790Z-85790Z-13.

Scheibe, P., Braumann, U.-D., Kuska, J.-P., Löffler, M., Simon, J. C., Paasch, U., and Wetzig, T. (2010). Image-processing chain for a three-dimensional reconstruction of basal cell carcinomas*. Experimental dermatology, 19(7):689-691.

Swartling, J., Pifferi, A., Enejder, A., and Andersson-Engels, S. (2003). Accelerated 
monte carlo models to simulate fluorescence spectra from layered tissues. J. Opt. Soc. Am. A, 20(4):714-727.

Valentine, R. (2011). Biological aspects of photodynamic therapy. Ph.D. Thesis, University of St Andrews.

Valentine, R., BRown, C., Moseley, H., Ibbotson, S., and Wood, K. (2011a). Monte carlo modeling of in vivo protoporphyrin ix fluorescence and singlet oxygen production during photodynamic therapy for patients presenting with superficial basal cell carcinomas. Journal of Biomedical Optics, 16(4):048002-048002-11.

Valentine, R. M., Ibbotson, S., Wood, K., Brown, C., and Moseley, H. (2013). Modelling fluorescence in clinical photodynamic therapy. Photochem. Photobiol. Sci., 12:203213.

Valentine, R. M., Ibbotson, S. H., Brown, C. T. A., Wood, K., and Moseley, H. (2011b). A quantitative comparison of 5-aminolaevulinic acid- and methyl aminolevulinate-induced fluorescence, photobleaching and pain during photodynamic therapy. Photochemistry and Photobiology, 87(1):242-249.

Van Gemert, M., Jacques, S., Sterenborg, H., and Star, W. M. (1989). Skin optics. Biomedical Engineering, IEEE Transactions on, 36(12):1146-1154.

Wachowska, M., Muchowicz, A., Firczuk, M., Gabrysiak, M., Winiarska, M., Wanczyk, M., Bojarczuk, K., and Golab, J. (2011). Aminolevulinic acid (ala) as a prodrug in photodynamic therapy of cancer. Molecules, 16(5):4140-4164.

Wang, L., Jacques, S. L., and Zheng, L. (1995). Mcmlmonte carlo modeling of light transport in multi-layered tissues. Computer methods and programs in biomedicine, 47(2):131-146.

Wilson, B. and Patterson, M. (2008). The physics, biophysics and technology of photodynamic therapy. Physics in Medicine and Biology, 53(9):R61.

Wood, K. (2013). http://www-star.st-and.ac.uk/ kw25/. Monte Carlo Radiation Transfer.

Wood, K., Haffner, L., Reynolds, R., Mathis, J. S., and Madsen, G. (2005). Estimating the porosity of the interstellar medium from three-dimensional photoionization modeling of $\mathrm{h}$ ii regions. The Astrophysical Journal, 633(1):295.

Wood, K. and R. J. Reynolds, R. (1999). A model for the scattered light contribution and polarization of the diffuse $\mathrm{h}$ galactic background. The Astrophysical Journal, $525(2): 799$.

Zhu, C. and Liu, Q. (2013). Review of monte carlo modeling of light transport in tissues. Journal of Biomedical Optics, 18(5):050902-050902. 